\title{
Community-Based Intervention Determines Tele-audiology Site Candidacy
}

\author{
Laura Coco, Au.D. ${ }^{1}$; Craig A. Champlin, Ph.D. ${ }^{1}$; Robert H. Eikelboom, Ph.D. ${ }^{2,3,4}$
}

${ }^{(1)}$ University of Texas at Austin, Austin, TX

${ }^{(2)}$ Ear Sciences Centre, The University of Western Australia, Nedlands, Australia

${ }^{(3)}$ Ear Science Institute Australia, Subiaco, Australia

${ }^{(4)}$ Department of Speech-Language Pathology and Audiology, University of Pretoria, Pretoria, South Africa

Purpose: Sections of the community face barriers to accessing audiology services. The aim of this study was to assess the barriers faced by people in typically underserved community settings and to provide audiology services in their natural environment. Information gathered by questionnaire was used to determine each site's candidacy as a potential tele-audiology site.

Methods: Sixty-three participants were recruited across three community sites that were identified as gathering places for individuals who experience barriers to accessing traditional clinical audiology services. Information about demographics and participant experience with barriers to access was gathered by a locally generated self-administered questionnaire. Pure-tone air-conduction audiometric exams were performed on participants with an automated portable diagnostic audiometer. Afterwards, the investigator provided counseling regarding hearing loss rehabilitation or hearing protection. Referrals were made when appropriate. 
Results: Pure tone averages were similar within sites, but varied across sites. At least $30 \%$ of individuals at each site reported they wanted to visit the audiologist more often. Each site reported different principal barriers to access, among them transportation, motivation and money. Eleven individuals were referred to the next level of care. Questionnaire results revealed special accommodations should be considered at each potential tele-audiology site.

Conclusion: The current study provided audiology services to individuals in their natural environment, identified many of the obstacles preventing individuals from pursuing traditional audiology services and provided information for the foundation of a tele-audiology practice.

Across the world, there is a disparity between the millions of individuals with a treatable hearing loss (WHO, 2008) compared to those who actually receive treatment (Margolis \& Morgan, 2008; Goulios \& Patuzzi 2008; Windmill \& Freeman, 2013). The traditional clinic-based model of care leaves many individuals unable to access services (WHO, 2010; Murphy, 2014). Some obstacles preventing access to care include high cost, lack of awareness of services, extra-audiological health priorities, as well as an unequal geographic distribution of hearing healthcare professionals in urban areas, isolating those in remote locations (McPherson, 2008). One way to address many of these obstacles is to decentralize the clinic and move towards a communitybased model, where services take place in the patient's natural environment (ILO, UNESCO \& WHO, 2004; Murphy, 2014). A community-based clinic not only reduces geographically isolated areas, benefitting individuals who lack transportation, it acknowledges the value of the social network on health improvement, promoting awareness of hearing healthcare within the community (Taylor, Braunack-Mayer, Cargo, Larkins \& Preston, 2013; Ataguba \& Mooney, 
2011). However, a global lack of audiologists (Goulios \& Patuzzi, 2008; Windmill \& Freeman, 2013) prevents these community-based clinics from being staffed by hearing healthcare professionals. Fortunately, tele-audiology, or the remote delivery of audiology services via communication technology, has proven a sustainable solution capable of connecting patients and professionals remotely over the Internet (Swanepoel et al., 2010; Swanepoel \& Hall, 2010). Locally staffed tele-audiology sites could connect virtually with an off-site audiologist at regular intervals for long-term support. Potential benefits to the remote delivery of services include reduced cost, reduced inconvenience, improved access and improved quality of care (Moffatt \& Eley, 2010).

However, before establishing the local patient site, or the tele-audiology "hub," it is important to gather information about the targeted community so services can be tailored to the their specific needs (Murphy, 2014). Previously, community-based health initiatives in medically underserved areas have demonstrated positive outcomes by implementing a model of care that is culturally appropriate (Ingram, M., Gallegos, G., \& Elenes, J., 2005; Staten et al., 2005). Conducting outreach in the community is a crucial first step before establishing a long-term health-based program.

In this study we provided hearing tests, as well as informational and rehabilitative counseling and referrals to individuals in a number of community settings that may be considered at risk of being underserved for audiology health care. Participants also completed a questionnaire that provided information about past experiences with audiology and barriers to accessing care. We then used the information in combination with other test results to assess barriers and determine the sites' candidacy as potential tele-audiology hubs. 


\section{METHOD}

A sample of 63 adults ( $43 \%$ female, $57 \%$ male) from 23 to 94 years of age $($ mean $=58, \mathrm{SD}=24)$ was recruited from three sites in Austin, Texas: a temporary shelter for immigrants and refugees, a residential retirement home and a government-assisted housing complex. These sites were identified as gathering places for individuals who are thought to have limited access to traditional audiological services due to the following predictor values: low socioeconomic status, non English-speakers, elderly, immobile, and individuals with other complicating health factors. Participants were recruited by flyer and word-of mouth, and were offered free earplugs and information about their hearing status in exchange for their participation.

Each session began with a brief oral case history, followed by otoscopy and a 10-item closed-set questionnaire that gathered information on the participant's demographics and past experience with hearing health-care. Next, pure-tone audiometry was performed with a KUDUwave audiometer (Figure 1) (GeoAxon, Pretoria, South Africa; http://www.emoyo.net/en/). This device has double attenuation transducers and passive noise monitoring, allowing diagnostic audiometry to be performed outside of the traditional sound treated room (Swanepoel, Matthysen, Eikelboom, Clark, \& Hall, 2015; Maclennan-Smith, Swanepoel \& Hall, 2013). The KUDUwave is computer-operated and, in the present study, was operated in automatic mode. The software presented pure-tones at irregular intervals, beginning with a tone at $40 \mathrm{~dB} \mathrm{HL}$ at $1 \mathrm{kHz}$ and continuing using a conventional 10-dB down, 5-dB up bracketing method (modified Hughson-Westlake) to determine the participant's softest threshold of hearing. After a threshold was obtained at $1 \mathrm{kHz}$, higher octave tones were tested before returning to lower frequencies. If the participant did not respond at the initial presentation level, the intensity was raised by $10 \mathrm{~dB}$ HL until a response was elicited and the $10 \mathrm{~dB}$ down $5 \mathrm{~dB}$ up method resumed. Air conduction 
thresholds did not require masking. Bone conduction audiometry was not included in this test battery. Instead, otoscopy and patient history was used to distinguish conductive from sensorineural loss. The Pure Tone Average (PTA, 0.5, 1, 2 and $4 \mathrm{kHz}$ ) for each ear was recorded.

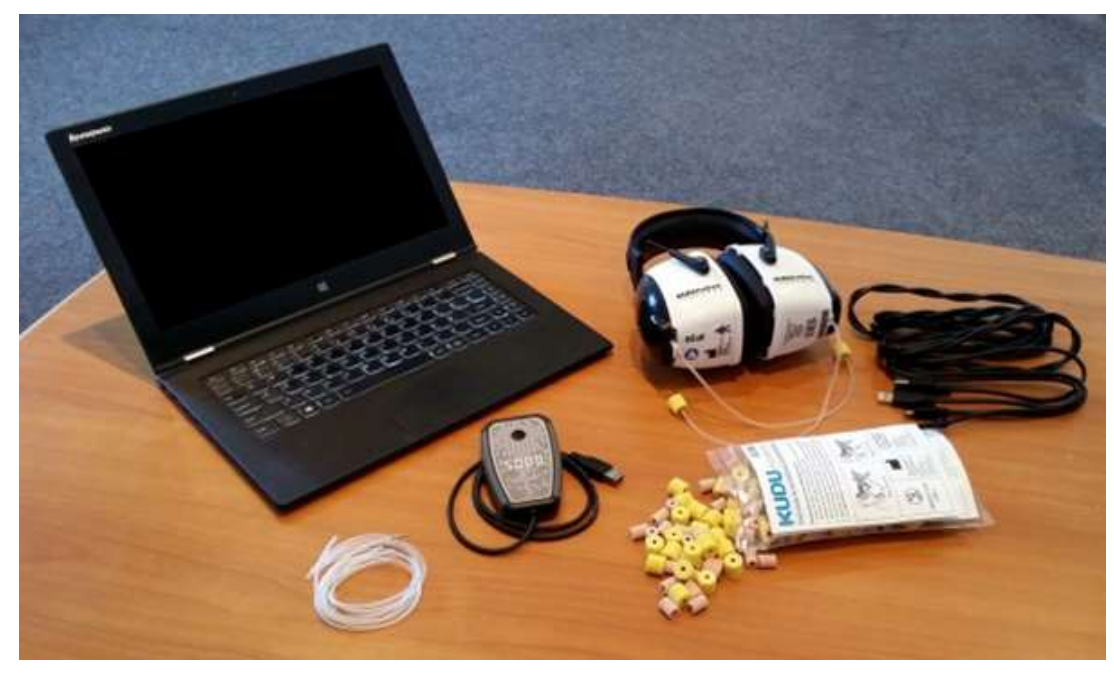

Fig. 1. KUDUwave 5000 Audiometer showing insert earphones, circumaural headphones, laptop computer and patient response button.

After the hearing test, the participant was engaged in a brief counseling session in which the results were discussed. All participants were given information about hearing loss and hearing protection. If otoscopy was remarkable or hearing loss was present, the participant was given information about next level of care and a referral list of local audiologists and otolaryngologists was provided.

All study procedures were reviewed and approved by the University of Texas Institutional Review Board, and each participant completed an informed consent process. Participants were not monetarily compensated for their time. 


\section{RESULTS}

Figure 2 summarizes hearing test results. Not surprisingly, with a mean age of 84 years, the greatest hearing loss was found in participants at the retirement home (mean PTA: $40.5 \mathrm{~dB}$ HL). At the low-income housing complex, the mean age was 48 years, and the mean PTA was $15.3 \mathrm{~dB}$ HL). With a mean age of 32 years, individuals at the temporary shelter in general had normal hearing (mean PTA: $11.8 \mathrm{~dB}$ HL). Most retirement home residents had previously seen an audiologist (88\%). However, $33 \%$ of these individuals reported they wished they could see an audiologist more often. Few residents of the temporary shelter had ever seen an audiologist (22\%), but many wanted to go more often (79\%). Only $26 \%$ of individuals at the low-income housing complex had ever seen an audiologist, but over half (60\%) indicated that they did want to.

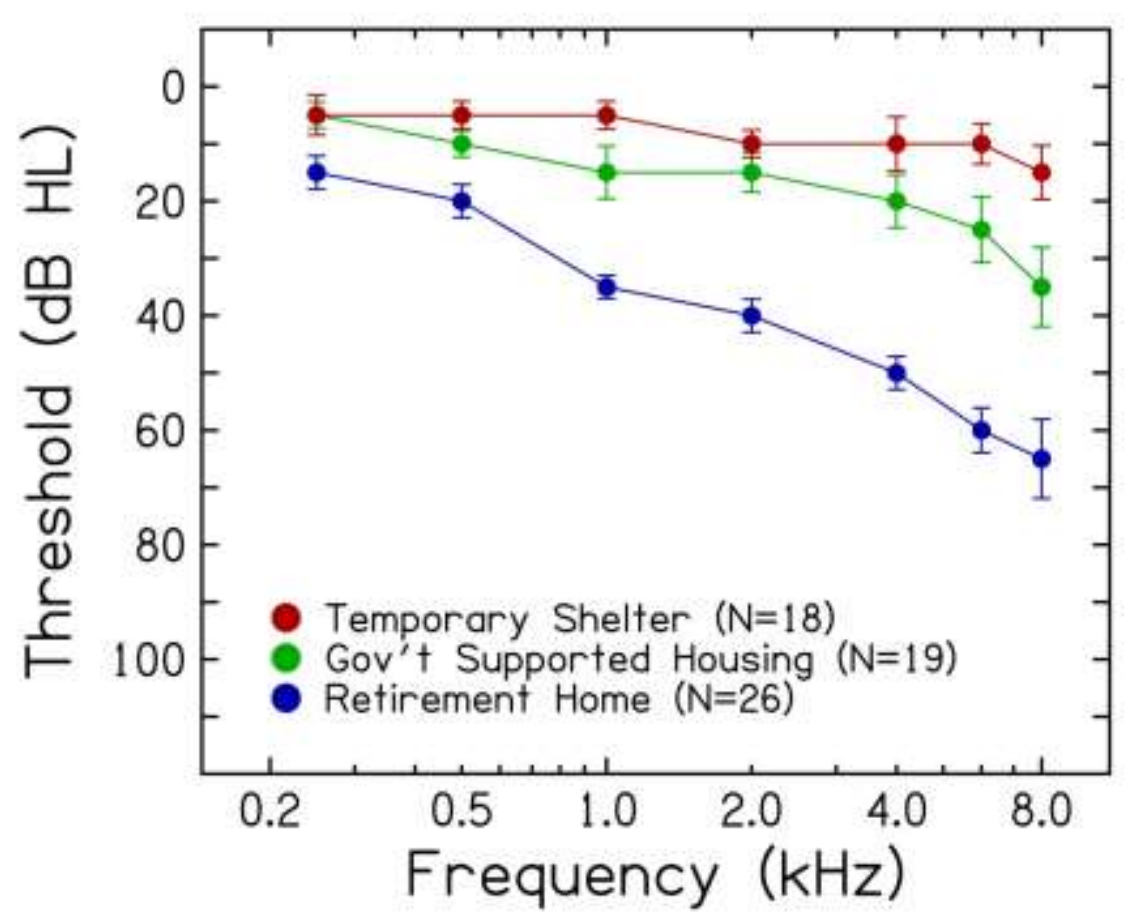

Fig 2. Mean Hearing Loss Thresholds (PTA) by Community Site. Error bars indicate +/- 1 standard deviation. 
When asked what prevented them from visiting the audiologist more often, responses varied between sites. For those at the retirement home, principal barriers to access were low motivation (62\%) and transportation (26\%). Those in the temporary shelter reported lack of money (54\%) and lack of knowledge of services (42\%). Finally, at the low-income housing complex, participants reported lack of knowledge of services (30\%) and low motivation (30\%). Across the three sites, a total of 11 participants were referred for the next level of care, 9 due to hearing loss and 2 for cerumen management. Results are summarized in Table 1.

Table 1: Demographics of participants and pre-hearing assessment survey results.

\begin{tabular}{|l|l|l|l|}
\hline & Temporary shelter & Low-income housing & Retirement home \\
\hline $\mathrm{n}$ & 18 & 19 & 26 \\
\hline Mean age (SD) in years & $32(9)$ & $48(11)$ & $84(7)$ \\
\hline Never visited an audiologist & $74 \%$ & $26 \%$ & $12 \%$ \\
\hline Wished to see an audiologist more \\
often & $79 \%$ & $60 \%$ & $33 \%$ \\
\hline Principal reported barriers to access & $\begin{array}{l}\text { lack of money (54\%) } \\
\text { lack of knowledge of }\end{array}$ & lack of knowledge of & low motivation \\
& services (42\%) & low motivation (30\%) & lack of \\
\hline
\end{tabular}

\section{DISCUSSION}

In this study, audiology services were provided to 63 individuals outside of the clinical environment. This community-based approach provided access to individuals, some of which 
experienced obstacles to accessing traditional hospital or clinic-based services. A locally generated questionnaire was used to collect demographic information, gauge past experiences with hearing healthcare and identify obstacles to accessing traditional services. Results from the hearing test and questionnaire provided crucial information when determining site candidacy as potential tele-audiology hubs.

This information gathering approach is in agreement with previous literature on communitybased intervention; to make services accessible and appropriate the intervention must adapt to the particular needs of the patient group (Murphy, 2014; WHO, 2010). Although the present study accomplished this by questionnaire, information can also be gathered by making affiliations with community leaders and other local public health organizations. Previously, a program targeting diabetes patients on the U.S.-Mexico border, demonstrated improved selfmanagement behaviors and clinical outcomes with a culturally appropriate model. In this community-based model, local collaborators called promotoras conducted outreach and patient education in a medically underserved region. Ingram and colleagues gathered feedback from participants by questionnaire and in-depth interview, adapting the program to the needs of the population (Ingram, M., Gallegos, G., \& Elenes, J., 2005). The current study was in line with this approach, although on a limited scale. Additional community-based audiology interventions are needed, especially in underserved regions.

The present study was a short-term intervention, and follow-up support was limited to a recommended list of local providers and services that could accommodate the patients with longterm support. However, especially in resource-poor populations, the risk of failed follow-up is high due to obstacles of transportation, money, or time (WHO, 2008). Therefore, the ideal community-based practice would be comprehensive and the local clinic's presence in the 
community would be long-term. Establishing a tele-audiology hub at each site offers a more sustainable solution. An off-site audiologist could provide long-term support to the site remotely, working in collaboration with a local facilitator. With the KUDUwave audiometer, diagnostic audiometry is automated, and can therefore be performed by locally trained staff. After the test, the audiologist could then connect with the patient to discuss results and counsel them on next steps. Additional services offered at the tele-audiology hub might include screening, diagnostic testing, as well as therapy and rehabilitation, including hearing aid adjustment and counseling (Swanepoel \& Hall, 2010). Establishing tele-audiology hubs at each site provides a bridge from each community to a hearing healthcare professional, increasing efficiency and minimizing barriers to access.

Although all three sites could likely benefit from tele-audiology support, services offered at each would differ. Individuals at the temporary shelter and low-income housing center had normal hearing, and therefore might benefit from a remotely operated monthly screening and education program. The primary candidates for a full-service tele-audiology hub might be the retirement home residents. The average individual there had a moderate degree of hearing loss and reported low motivation as one of their primary obstacles to accessing audiology services. For that reason, a tele-audiology hub located within the retirement home, facilitated by locally trained staff, might encourage residents to take action. This hub could include a variety of services, including diagnostic tests, hearing aid fittings, and long-term follow-up care. A continuation of this study may include the implementation and program evaluation of these tele-audiology pilot sites.

Additional factors related to the success of potential tele-audiology pilot sites include basic technological infrastructure required to facilitate services. In addition, the socioeconomic status and comorbid medical conditions of the individuals targeted must be taken into account, as this 
could influence the uptake of services. Future research is needed to evaluate the effects of these factors on a tele-audiology pilot program.

\section{CONCLUSION}

This project provided audiology services to individuals within their community and gathered information about the barriers that might prevent them from accessing traditional clinic-based services. Information helped determine candidacy for potential teleaudiology pilot sites.

\section{ACKNOWLEDGEMENTS}

The investigators thank the Oticon Foundation for the donation of the KUDUwave Audiometer. Part of this work was presented at the Second International Meeting on Internet \& Audiology, Helsingør, Denmark, September 24-25, 2015.

\section{REFERENCES}

Ataguba, J. E., \& Mooney, G. (2011). A communitarian approach to public health. Health Care Analysis, 19(2), 154-164.

Goulios, H., \& Patuzzi, R. B. (2008). Audiology education and practice from an international perspective. International Journal of Audiology, 47(10), 647-664. 
ILO, UNESCO, WHO. (2004). Community-Based Rehabilitation: A Strategy for Rehabilitation, Equalization of Opportunities, Poverty Reduction and Social Inclusion of People with Disabilities. Joint position paper. Retrieved November 20, 2015 from http://apps.who.int/ iris/bitstream/10665/43060/1/9241592389_eng.pdf

Ingram, M., Gallegos, G., \& Elenes, J. (2005). Diabetes is a community issue: the critical elements of a successful outreach and education model on the US-Mexico border. Prev Chronic Dis, 4(4).

Maclennan-Smith, F., Swanepoel, D., \& Hall, J.W., III. (2013). Validity of diagnostic pure-tone audiometry without a sound-treated environment in older adults. International Journal of Audiology, 52, 66-73.

Margolis, R. H., \& Morgan, D. E. (2008). Automated pure-tone audiometry: an analysis of capacity, need, and benefit. American Journal of Audiology, 17(2), 109-113.

McPherson, B. (2008). Audiology in developing countries. New York: Nova Science Publishers, Inc.

Murphy, J. (2014). Community Based Interventions: Philosophy and Action. New York: Springer Science.

Moffatt, J. J., \& Eley, D. S. (2010). The reported benefits of telehealth for rural Australians. Australian Health Review, 34(3), 276-281.

Staten, L.K., Scheu, L. L., Bronson, D., Peña, V., Elenes, J. (2005). Pasos Adelante: The Effectiveness of a Community-based Chronic Disease Prevention Program. 
Swanepoel, D. W., \& Hall, J. W., III. (2010). A systematic review of telehealth applications in audiology. Telemedicine and e-Health, 16(2), 181-200.

Swanepoel, D.W., Matthysen, C., Eikelboom, R.H., Clark, J.L., \& Hall, J.W., III. (2015). Puretone audiometry outside a sound booth using earphone attenuation, integrated noise monitoring, and automation, International Journal of Audiology, 54, (11) 777-785.

Taylor, J., Braunack-Mayer, A., Cargo, M., Larkins, S., \& Preston, R. (2013). A role for communities in primary prevention of chronic illness? Case studies in regional Australia. Qualitative Health Research, 23(8), 1103-1113.

Windmill, I. M., \& Freeman, B. A. (2013). Demand for Audiology Services: 30-Yr Projections and Impact on Academic Programs. Journal of the American Academy of Audiology, 24(5), 407416.

World Health Organization (2008). The global burden of disease: 2004 update. Geneva: World Health Organization. Retrieved October 15, 2015 from http://www.who.int/healthinfo/global_bu rden_disease/GBD_report_2004update_full.pdf

World Health Organization (2010). Community based rehabilitation - CBR guidelines. WHO, UNESCO, ILO and IDDC, Geneva, Switzerland. Retrieved October 1, 2015 from http://apps.who.int/iris/bitstream/10665/44405/9/9789241548052_introductory_eng.pdf 